\title{
The Benefits of Exercise Effect on Cancer: A Review
}

\author{
Namju Lee \\ Institute of Information Technology, Kwangwoon University, Seoul, Korea
}

PURPOSE: Exercise has been greatly shown to be effective on cancer patients and thus improves functional capacity in cancer patients and lowers a risk of cancer recurrence. The growing numbers of cancer population has been diagnosed and therefore, effect of exercise on cancer should be focused as a great intervention. Therefore, this study reviews the benefits of exercise on cancer and exercise guidelines for cancer population.

METHODS: This study reviews 30 previous studies focusing on the benefits of exercise on various types of cancer such as breast, colon, colorectal, liver, lung, ovary, brain, prostate, and melanoma.

RESULTS: Improvement in muscle strength, aerobic capacity, body balance throughout optimal exercise would be necessary for cancer patients and survivors for their life-long health by improving overall body function. To reach a healthy life, they need to conduct at least 150 minutes of moderate aerobic exercise or 75 minutes of vigorous aerobic exercise per week, and also at least 2 days per week of resistance exercise with moderate to high intensity for all major muscle groups. Exercise intervention needs to be safe during and after cancer treatments and results in improvements in physical functioning, cancer-related symptoms, and quality of life. Especially, patients and/or survivors with prostate cancer and melanoma are required to exercise with prescription because exercise may induce unexpected effects on health in those population.

CONCLUSIONS: Implications for exercise affects related to cancer are still unknown; however, the benefits of exercise to physical functioning and quality of life are sufficient for cancer patients and survivors.

Key words: Exercise, Cancer, Intervention, Exercise guideline, Adaptation

\section{INTRODUCTION}

Previous studies proved that exercise has impact on health and wellbeing of humans, which is associated with a reduced risk of mortality in life [1]. Moreover, exercise has been greatly shown to be effective on cancer patients and thus improves functional capacity in cancer patients and lowers a risk of cancer recurrence such as breast cancer and colorectal cancer by 30-60\% [2-5]. However, optimal timing and type of exercise and exercise mechanism on cancer have not been clearly identified although there is an impact of exercise on various types of cancer such as breast, colon, colorectal, liver, lung, ovary, brain, prostate, and melanoma $[3,6]$.
Exercise has been considered to be a low-cost and safe intervention for cancer patients and survivors having beneficial effects on body functions and health-related fitness including cardiovascular fitness, muscle fitness, body composition, mental and emotional health, and quality of life [7-11]. Moreover, exercise is associated with overall physical changes and the magnitude of such changes is influenced by the intensity and duration of exercise performed [6]. For cancer survivors, exercise has been known to be beneficial for reducing fatigue, weakness, pain, and improving functional ability [4]. To date, hundreds of prospective studies have examined associations between physical activity and cancer risk; however, study results have been inconclusive for most cancer types because of the small case numbers and physical activity types.

Corresponding author: Namju Lee Tel +82-2-940-5114 Fax: +82-2-917-6147 E-mail namju1210dgmail.com

Received 5 Oct 2019 Revised 26 Oct 2019 Accepted 1 Nov 2019

@) This is an Open Access article distributed under the terms of the Creative Commons Attribution Non-Commercial License (https://creativecommons.org/licenses/by-nd/4.0// which permits unrestricted non-commercial use, distribution, and reproduction in any medium, provided the original work is properly cited. 
The growing numbers of cancer population has been diagnosed and therefore, the effect of exercise on cancer should be focused as a great intervention. It has been well known that cancer treatment causes reduced physical function and impaired quality of life and these negative aspects have been reported by surgery, radiation therapies, hormonal treatment, and targeted therapies $[12,13]$. Especially, cancer survivors should avoid inactivity in their life and need to be adapted to exercise habit. Hence, a growing understanding of the benefits of exercise for cancer over the recent years has been payed attention to exercise professionals and researchers as well as cancer patients and survivors. While many good effects of exercise on cancer are well known in previous studies, it is necessary to reconsider and pay attention to the relationship between exercise and cancer. Therefore, this study focuses the benefits of exercise on cancer and exercise guidelines for cancer patients and survivors as well as healthcare professionals, although exercise mechanism effect on various types of cancers has not been clearly proved yet.

\section{EFFECTS OF EXERCISE ON CANCER}

Over several decades, strong evidence has been found to support the positive efficacy of exercise for cancer patients and survivors. It has been reported that exercise mediates changes in body composition, hormone levels, systemic inflammation, immune cell function, and so on [6]. Among those changes, exercise-induced immune cell function related to cancer needs to be discussed. Natural killer cells (NK cells) are known as killing cancer cells [14] and they are known to be the most sensitive immune cells responded to exercise [15]. Exercise leads an increased cardiac output and induces a rapid increase in blood counts of immune cells [16]. It has been addressed that NK cells are increased after 30 minutes of aerobic exercise and prolonged training does not lead to increased NK cells; however, maximal level of NK cells can be maintained by 3 hours [15], which can evoke positive effect on cancer.

Moreover, NK cells is related to interleukin-6 (IL-6) and IL-6 is one of the myokines from the contracting muscles during exercise [17,18]. During exercise, exercise-engaged muscles rapidly released IL- 6 and the plasma level of IL-6 is increased, which plays a role to increase mobilization of NK cells. A previous study [3] reported that leisure time physical activity is significantly associated with a lower risk of cancer occurred in kidney, lung, colon, liver, and breast. Another study [19] investigated natural cytotoxic activity of peripheral-blood lymphocytes and cancer incidence with an 11-year follow-up study of a general population. Natural cyto- toxic activity of peripheral-blood mononuclear cells was assessed in 3,625 residents of a Japanese population mostly older than 40 years of age and they used 154 cancer cases. They found that a high level of cytotoxic activity among peripheral blood lymphocytes was associated with a lower risk of cancer, which shows NK cells are active in early stage of the cancer. They concluded that medium and high cytotoxic activity of peripheral-blood lymphocytes is associated with reduced cancer risk. Therefore, NK cells is suggested as a cancer protection role rather than cancer treatment and they may be a link between regular physical activity and overall health status in human body [14].

Another NK cell related study [20] investigated how running exercise suppresses tumor growth through epinephrine- and IL-6-dependent NK cell mobilization and redistribution. They randomized mice with tumor to perform voluntary wheel running and they reported that there was an over $60 \%$ reduction in tumor incidence of mice. They explained the mechanism of exercise on cancer as follows; NK cells were mobilized by epinephrine, and blockade of $\beta$-adrenergic signaling blunted trainingdependent tumor inhibition and epinephrine induced a selective mobilization of IL-6-sensitive NK cells, which induced IL-6-blocking antibodies blunted training-induced tumor suppression, intratumoral NK cell infiltration, and NK cell activation. They concluded that exercise, epinephrine, and IL-6 to NK cell mobilization and redistribution has a link to control of tumor growth. Therefore, it seems that exercise can mobilize NK cells associated with IL-6, which is able to kill cancer cells and/or prevent cells from cancer in human body.

Most previous studies proved that exercise is essential for preventing and treating various types of cancers in humans; however, one previous study [3] investigated that prostate cancer and melanoma showed a higher risk in physically active population. They recruited a total of 1.44 million participants including 26 types of cancer and found that leisuretime physical activity was associated with lower risks of many cancer types except above 2 types of cancer. They assumed that greater sun exposure seemed to be the likely reason for the increase in melanoma risk, as physical activity is frequently done outdoors in light clothing and has been associated with increased risk of sun burn. In addition, they explained that physically active men are more likely than inactive men to receive digital rectal exams and/or prostate-specific antigen screening, which increases the likelihood of diagnosing indolent prostate cancers and thus the positive association of exercise and increased cancer risk they observed could therefore be due to screening bias. On the contrary, there is a study resulting that physical activity was associated with lower 
overall mortality and prostate cancer mortality. Kenfield and colleagues [21] evaluated physical activity in relation to overall and mortality among 2,705 prostate cancer survivors. They concluded that vigorous activity such as biking, tennis, jogging, and swimming for over 3 hours per week may substantially improve prostate cancer-specific survival, which shows regular exercise has the same effect on prostate cancer as it has on other types of cancer. In addition, Ibrahim and Al-Homaidh [22] investigated the role of exercise on breast cancer using previously published researches by meta-analysis because the outcome has been controversial with inconsistent data. The study analysis showed that post-diagnosis exercise reduced breast cancer deaths and all causes mortality among patients with estrogen receptor-positive tumor, while women with estrogen receptor-negative disease showed no gain. Although there was no exercise effect on estrogen receptor-negative breast cancer women, they concluded that appropriate exercise should also be conducted for breast cancer survivors. Based on the various previous study results, exercise may be an efficient way to treat various types of cancer and prevent reoccurrence cancer in cancer patients and survivors. Moreover, exercise intervention would be preventive and/or therapeutic for cancer patients and survivors and cancer related time periods would be very important for them. Optimal timing exercise intervention needs to be guided for them and thus, the physical activity and cancer control frame work is shown in Figure 1.

\section{EXERCISE GUIDELINES FOR CANCER}

For reviewing exercise and cancer research and developing exercise guidelines, the American College of Sports Medicine (ACSM) summoned various groups of experts in 2009 because most clinicians historically advised cancer patients to rest and to avoid activity at those times and the ACSM challenged those recommendation [12]. The ACSM tried to provide the safety and efficacy of exercise training during and after adjuvant cancer therapy and they recommended that all cancer survivors need to maintain at least 150 minutes of moderate aerobic exercise or 75 minutes of vigorous aerobic exercise per week and also recommended that at least 2 days per week of resistance exercise with moderate to high intensity for all major muscle groups [12].

Exercise seems to be a valuable for cancer protection because previous studies proved that 50 percent of cancer survivors conducted exercise among 3 out of 4 most common cancers [21-25]. Meyerhardt and colleagues [23] investigated the influence of exercise on colorectal cancer survivors. They conducted an observational study of 573 women with stage 1 to 3 colorectal cancer and concluded that increasing levels of exercise after diagnosis of nonmetastatic colorectal cancer reduced cancer-specific mortality $(P$ for trend $=.008)$ and overall mortality $(P$ for trend $=.003)$. They also compared with women who engaged in less than 3 metabolic equivalent task (MET)-hours per week of physical activity, those engaging in at least 18 MET-hours per week had an adjusted hazard ratio for colorectal cancer-specific mortality of 0.39 (95\% CI, 0.18 to 0.82$)$ and an adjusted hazard ratio for overall mortality of $0.43(95 \%$ CI, 0.25 to 0.74 ). They proved that physical activity could reduce the risk of colorectal cancer-specific and overall mortality. Another study [24] observed 45 published researches and reported that there was consistent evidence from 27 observational studies that physical activity is associated with reduced all-cause, breast cancer-specific, and colon cancerspecific mortality. Also they mentioned that randomized controlled trials of exercise suggested that exercise may result in beneficial changes in the level of insulin, insulin-related pathways, inflammation, and immunity. A prospective observational study [25] recruited 832 patients with stage 3 colon cancer enrolled in a randomized adjuvant chemotherapy

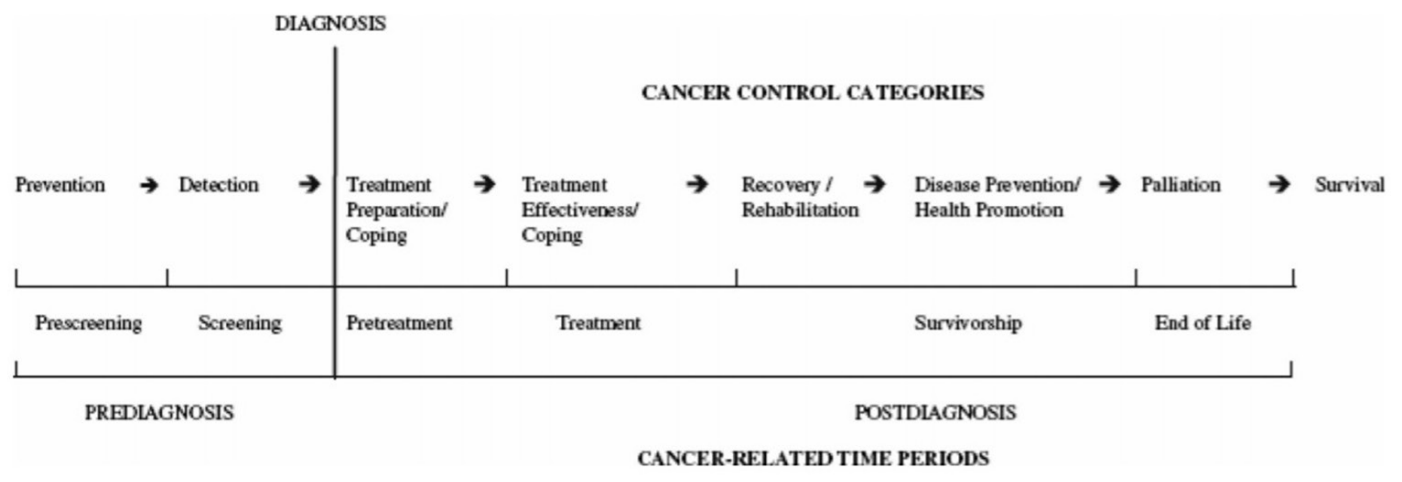

Fig. 1. Physical activity and cancer control frame work (reprinted from Schmitz et al., 2010 [12]). 
trial and the patients participated in the study reported on various recreational physical activities approximately 6 months after completion of therapy. The researchers compared with patients engaged in less than 3 MET-hours per week of physical activity, the adjusted hazard ratio for disease-free survival was 0.51 (95\% CI, 0.26 to 0.97 ) for 18 to 26.9 METhours per week and 0.55 (95\% CI, 0.33 to 0.91 ) for 27 or more METhours per week. Postdiagnosis activity was associated with similar improvements in recurrence-free survival $(P$ for trend $=.03$ ) and overall survival ( $P$ for trend $=.01$ ). They mentioned that they proved a positive physical activity effect by reducing the risk of cancer recurrence and mortality, approximately 6 months after adjuvant chemotherapy.

Once several previous studies have demonstrated that regular exercise has a positive effect on different types of cancer, it is now necessary to know how to exercise is effective for the treatment and/or prevention of cancer. A recent study [26] investigated the benefits of exercise during and following cancer treatments to determine exercise intervention evidence into practice. Participants were 2,500 cancer survivors and conducted 60 minutes of mild to moderate intensity full body exercise twice per week for 12 weeks. They found the primary effectiveness outcome is the proportion of participants meeting or exceeding 150 minutes of moderate intensity exercise per week at 1-year follow up. They also addressed that community-based exercise program for cancer survivors should include 5 stages as follows; the reach stage for individual goal, the effectiveness stage for individual and institutional level, the adoption stage for institutional level, the implementation stage for community, and lastly, the maintenance stage for individual, institutional, and community. They suggested that there were advantages of community-based programs, which includes high accessibility, safety and supervision of exercise and social interaction [27].

Another recent study [4] proved that the amount of activity required to achieve protective benefits of exercise for cancer patients and survivors is moderate (e.g., walking 30 minutes per day at 4 kilometers per hour). They emphasized that cancer treatment-specific side effects, such as anxiety, depression, fatigue, hair loss, nausea, peripheral neuropathy, pain, skin rashes, vomiting, and changes in self-esteem and body image, have a negative impact on quality of life and contribute to the declines in physical and emotional function as well. Therefore, the goal is to adapt exercise and to give the cancer patients and survivors the optimal exercise benefits, which is necessary for the quality of life. In addition, Luan and colleagues [28] addressed that cancer is a major cause of death in the United States and causes one-quarter of all death [29] and they analyzed
Table 1. Types of exercise intervention for breast, colon, lung, and prostate cancers

\begin{tabular}{lc}
\hline \multicolumn{1}{c}{ Types of exercise } & Cancers \\
\hline Aerobic exercise & Colon, Lung, Prostate \\
Resistance exercise & Breast, lung, Prostate \\
$\begin{array}{l}\text { Combined aerobic exercise with } \\
\text { resistance training }\end{array}$ & Breast, Colon, Lung, Prostate \\
High-intensity interval training & Lung \\
\hline
\end{tabular}

previous researches suggesting that combined aerobic exercise with resistance training for breast, colon, lung, and prostate cancer. They also suggested that exercise prescription for colon, lung, and prostate cancer would include moderate- and high-intensity of aerobic and resistance exercise. For breast cancer, they suggested from low to moderate exercise intensity unlike other kinds of cancer similar as previous studies [3,22]. Travier and colleagues [30] also mentioned that moderate intensity of aerobic exercise combined with resistance exercise benefited early adjuvant breast cancer treatment. They conducted 18 weeks of an exercise program and there was a positive effect on physical fatigue, cardiopulmonary function, and muscle strength in breast cancer patients. Several types of exercise intervention and their uses in the treatment for breast, colon, lung, and prostate cancers are shown in Table 1.

Based upon previous exercise guidelines, improvement in muscle strength, aerobic capacity, body balance throughout optimal exercise program would be necessary for cancer patients and survivors for their life -long health by improving overall body function. To reach a healthy life, they need to conduct at least 150 minutes of moderate aerobic exercise or 75 minutes of vigorous aerobic exercise per week and also at least 2 days per week of resistance exercise with moderate to high intensity for all major muscle groups.

\section{CONCLUSION}

Exercise intervention for cancer patients and survivors needs to be safe during and after cancer treatments and to result in improvements in physical functioning, cancer-related symptom, and quality of life. Implications for clear exercise affects related to cancer are still unknown; however, the benefits of exercise to physical functioning and quality of life are sufficient for the recommendation with specific exercise programming adaptations. Improvement in muscle strength, aerobic capacity, body balance throughout optimal exercise program would be necessary for cancer patients and survivors for their life-long health by improving 
overall body function. To reach a healthy life, they need to conduct at least 150 minutes of moderate aerobic exercise or 75 minutes of vigorous aerobic exercise per week and also at least 2 days per week of resistance exercise with moderate to high intensity for all major muscle groups. In addition, longitudinal studies related to exercise and cancer would be necessary to better understand specific exercise mechanism on cancer prevalence.

\section{CONFLICT OF INTEREST}

No potential conflict of interest relevant to this article was reported.

\section{AUTHOR CONTRIBUTION}

One author should have made substantial contributions to all of the following: (1) N. Lee contributed the conception and design of the study, (2) N. Lee contributed the drafting the article or revising it critically for important intellectual content, and (3) final approval of the version to be submitted. One author edited, revised and approved final version of manuscript.

\section{ORCID}

Namju Lee https://orcid.org/0000-0002-2567-5608

\section{REFERENCES}

1. Kujala UM, Kaprio J, Sarna S, Koskenvuo M. Relationship of leisuretime physical activity and mortality: the Finnish twin cohort. JAMA. 1998;279(6):440-4.

2. Ballard-Barbash R, Friedenreich CM, Courneya KS, Siddiqi SM, McTiernan A et al. Physical activity, biomarkers, and disease outcomes in cancer survivors: a systematic review. J Natl Cancer Inst. 2012;104(11): $815-40$.

3. Moore SC, Lee IM, Weiderpass E, Campbell PT, Sampson JN, et al. Association of leisure-time physical activity with risk of 26 types of cancer in 1.44 million adults. JAMA Intern Med. 2016;176(6):816-25.

4. Schwartz AL, de Heer HD, Bea JW. Initiating Exercise Interventions to Promote Wellness in Cancer Patients and Survivors. Oncology (Williston Park). 2017;31(10):711-7.

5. Irwin ML, McTiernan A, Manson JE, Thomson CA, Sternfeld B, et al.
Physical activity and survival in postmenopausal women with breast cancer: results from the women's health initiative. Cancer Prev Res (Phila). 2011;4(4):522-9.

6. Idorn M, Thor Straten P. Exercise and cancer: from "healthy" to "therapeutic"? Cancer Immunol Immunother. 2017;66(5):667-71.

7. Cormie P, Zopf EM, Zhang X, Schmitz KH. The Impact of Exercise on Cancer Mortality, Recurrence, and Treatment-Related Adverse Effects. Epidemiol Rev. 2017;39(1):71-92.

8. Rock CL, Doyle C, Demark-Wahnefried W, Meyerhardt J, Courneya KS, et al. Nutrition and physical activity guidelines for cancer survivors. CA Cancer J Clin. 2012;62(4):243-74.

9. Speck RM, Courneya KS, Mâsse LC, Duval S, Schmitz KH. An update of controlled physical activity trials in cancer survivors: a systematic review and meta-analysis. J Cancer Surviv. 2010;4(2):87-100.

10. Lancaster GI, Febbraio MA. The immunomodulating role of exercise in metabolic disease. Trends Immunol. 2014;35(6):262-9.

11. Fabbrini E, Sullivan S, Klein S. Obesity and nonalcoholic fatty liver disease: biochemical, metabolic, and clinical implications. Hepatology 2010;51(2):679-89.

12. Schmitz KH, Courneya KS, Matthews C, Demark-Wahnefried W, Galvão DA, et al. American College of Sports Medicine roundtable on exercise guidelines for cancer survivors. Med Sci Sports Exerc. 2010; 42(7):1409-26

13. Jones LW, Eves ND, Haykowsky M, Freedland SJ, Mackey JR. Exercise intolerance in cancer and the role of exercise therapy to reverse dysfunction. Lancet Oncol. 2009;10(6):598-605.

14. Kärre K, Ljunggren HG, Piontek G, Kiessling R. Selective rejection of H-2-deficient lymphoma variants suggests alternative immune defence strategy. Nature. 1986;319(6055):675-8

15. Timmons BW, Cieslak T. Human natural killer cell subsets and acute exercise: a brief review. Exerc Immunol Rev. 2008;14:8-23.

16. Walsh NP, Gleeson M, Shephard RJ, Gleeson M, Woods JA, et al. Position statement. Part one: immune function and exercise. Exerc Immunol Rev. 2011;17:6-63.

17. Cullen T, Thomas AW, Webb R, Hughes MG. Interleukin-6 and associated cytokine responses to an acute bout of high intensity interval exercise: the effect of exercise intensity and volume. Appl Physiol Nutr Metab. 2016;41(8):803-8.

18. Steensberg A, Keller C, Starkie RL, Osada T, Febbraio MA, et al. IL-6 and TNF-alpha expression in, and release from, contracting human skeletal muscle. Am J Physiol Endocrinol Metabol. 2002;283(6):E1272- 


\section{E1278.}

19. Imai K, Matsuyama S, Miyake S, Suga K, Nakachi K. Natural cytotoxic activity of peripheral-blood lymphocytes and cancer incidence: an 11-year follow-up study of a general population. Lancet. 2000; 356(9244):1795-9.

20. Pedersen L, Idorn M, Olofsson GH, Lauenborg B, Nookaew I, et al. Voluntary Running Suppresses Tumor Growth through Epinephrineand IL-6-Dependent NK Cell Mobilization and Redistribution. Cell Metabol. 2016;23(3):554-62.

21. Kenfield SA, Stampfer MJ, Giovannucci E, Chan JM. Physical activity and survival after prostate cancer diagnosis in the health professionals follow-up study. J Clin Oncol. 2011;29(6):726-32.

22. Ibrahim EM, Al-Homaidh A. Physical activity and survival after breast cancer diagnosis: meta-analysis of published studies. Med Oncol. 2011;28(3):753-65.

23. Meyerhardt JA, Giovannucci EL, Holmes MD, Chan AT, Chan JA, et al. Physical activity and survival after colorectal cancer diagnosis. J Clin Oncol. 2006;24(22):3527-34.

24. Ballard-Barbash R, Friedenreich CM, Courneya KS, Siddiqi SM, McTiernan A, et al. Physical activity, biomarkers, and disease outcomes in cancer survivors: a systematic review. J Natl Cancer Inst. 2012;104(11): $815-40$.
25. Meyerhardt JA, Heseltine D, Niedzwiecki D, Hollis D, Saltz LB, et al Impact of physical activity on cancer recurrence and survival in patients with stage III colon cancer: findings from CALGB 89803. J Clin Oncol. 2006;24(22):3535-41.

26. McNeely ML, Sellar C, Williamson T, Shea-Budgell M, Joy AA, et al. Community-based exercise for health promotion and secondary cancer prevention in Canada: protocol for a hybrid effectiveness-implementation study. BMJ Open. 2019;9(9):e029975.

27. Santa Mina D, Au D, Brunet J, Jones J, Tomlinson G, et al. Effects of the community-based Wellspring cancer exercise program on functional and psychosocial outcomes in cancer survivors. Curr Oncol. 2017; 24(5):284-94.

28. Luan X, Tian X, Zhang H, Huang R, Li N, et al. Exercise as a prescription for patients with various diseases. J Sport Health Sci. 2019;8(5): $422-41$

29. Siegel R, Ma J, Zou Z, Jemal A. Cancer statistics, 2014. CA Cancer J Clin. 2014;64(1):9-29.

30. Travier N, Velthuis MJ, Steins Bisschop CN, van den Buijs B, Monninkhof EM, et al. Effects of an 18-week exercise programme started early during breast cancer treatment: a randomised controlled trial. BMC Med. 2015;13:121. 